Pacific Journal of Mathematics

P PROXIMATION AND INTERPOLATION FOR SOME SPACES 


\section{APPROXIMATION AND INTERPOLATION FOR SOME SPACES OF ANALYTIC FUNCTIONS IN THE UNIT DISC}

\section{A. STRAY}

Let $U$ be a bounded open subset of the complex plane $C$ such that $U$ and $C \backslash \bar{U}$ are connected. (If $B \subset C, \bar{B}$ denotes its closure in C.) $H^{\infty}(U)$ is the space of all bounded analytic functions defined on $U$. Let $S \subset U$ be the zero set of a nonzero function in $H^{\infty}(U)$.

Necessary and sufficient conditions on $S$ are given for the existence of an open set $0 \supset \bar{U} \backslash(\bar{S} \backslash S)$ such that $H^{\infty}(0)$ and $H^{\infty}(U)$ have the same restrictions to $S$. If $U$ is the unit disc $D=$ $\{z:|z|<1\}$ and $S$ is as above, the following result holds for all the Hardy spaces $H^{p}(D), 0<p \leq \infty$ : Given $g \in H^{p}(D)$, there is a function $f$ analytic in $C \backslash(\bar{S} \backslash S)$ such that $\left.f\right|_{D} \in H^{P}(D)$ and $f=g$ on $S$.

If $S$ and $U$ are as above, $\left.H^{\infty}(U)\right|_{S}$ denotes the set of restrictions $\left.f\right|_{S}$ of all $f \in H^{\infty}(U)$. If $S=\left\{z_{n}\right\} \subset D$ satisfies $\Sigma_{n}\left(1-\left|z_{n}\right|\right)<\infty$, Detraz [3] proved the following result

$\left(^{*}\right): \quad$ There exists an open set $0 \supset \bar{D} \backslash(\bar{S} \backslash S)$ such that

$$
\left.H^{\infty}(0)\right|_{s}=\left.H^{\infty}(D)\right|_{s} .
$$

In this paper we give two extensions of this result. First we show that $\left(^{*}\right)$ holds for domains of a somewhat more general type than the unit disc $D$. Consider the following statement which is very similar to $\left(^{*}\right)$ :

(**) There exists an open set $V$ such that $\bar{V} \backslash(\bar{S} \backslash S) \subset D$ and

$$
\left.H^{\infty}(V)\right|_{s}=\left.H^{\infty}(D)\right|_{s} .
$$

It turns out that conditions $\left({ }^{*}\right)$ and $\left({ }^{* *}\right)$ are equivalent, even with $D$ replaced by a somewhat more general set.

We shall make some use of the theory of the classical $H^{p}$ spaces. We refer to [4] or [9] in this connection. Before stating our first result, we mention some more notation. If $f$ is a complex valued function defined for each $z \in B$ we put $\|f\|_{B}=\sup \{|f(z)|, z \in B\}$. If $U \subset C$ is open, $H^{\infty}(U)$ is a Banach algebra with sup norm on $U$ and we denote by $M$ the maximal ideal space of $H^{\infty}(U)$. The maximal ideals $m \in M$ are identified with the multiplicative functionals on $H^{\infty}(U)$ they correspond to. If $S \subset U$ is relatively closed and $I$ denotes the set of all $f \in H^{\infty}(U)$ which are zero on 
$S$, we define $\tilde{S}=\{m \in M: m(f)=0 \quad f \in I\}$. (Cf. p. 345 in [3]). We have a projection $\Pi: M \rightarrow \bar{U}$ given by $m \rightarrow m(e)$ where $e \in H^{\infty}(U)$ is the function $z \rightarrow z$. For a detailed study of $M$ we refer to [7] and Ch. 10 in [9]. Other results like $\left(^{*}\right)$ can be found in [1], [5], [8], [11] and [14].

With the notation as above we now state:

THEOREM 1. Let $U$ be the interior of a compact set $X$ and assume both $U$ and $\mathbf{C} \backslash X$ are connected. If $S \subset U$ is the zero set of a nonzero function in $H^{\infty}(U)$, the following statements are equivalent:

(i) There exists an open set $0 \supset \bar{U} \backslash(\bar{S} \backslash S)$ such that $\left.H^{\infty}(0)\right|_{S}=$ $\left.H^{\infty}(U)\right|_{S}$

(ii ) There exists an open set $V$ such that $S \subset V \subset U, \bar{V} \backslash(\bar{S} \backslash S) \subset U$ and $\left.H^{\infty}(V)\right|_{s}=\left.H^{\infty}(U)\right|_{S}$

(iii) $\Pi(\tilde{S}) \subset \bar{S}$.

REMARK. The author is indebted to the referee for an example where (iii) fails. For details of this example see the final remarks. If the boundary $\partial U$ of $U$ is a Jordan arc, it is easy to verify that (iii) holds, but considerably weaker conditions on $\partial U$ also imply (iii).

Proof: If $\bar{S} \supset \partial U$, the theorem trivially holds with $0=V=U$. Assume now $(\partial U) \backslash \bar{S} \neq \varnothing$. We prove the implications (ii) $\Rightarrow$ (i), (i) $\Rightarrow$ (iii) and (iii) $\Rightarrow$ (ii). We assume first that (ii) is true and consider the restriction map $R:\left.H^{\infty}(0) \rightarrow H^{\infty}(V)\right|_{S}$ where $0 \supset \bar{U} \backslash(\bar{S} \backslash S)$ is some open set and where $\left.H^{\infty}(V)\right|_{S}$ has the quotient norm induced from $H^{\infty}(V)$. We need to prove that $R$ maps $H^{\infty}(0)$ onto $\left.H^{\infty}(V)\right|_{S}$. It is sufficient to find constants $L>0$ and $\epsilon \in(0,1)$ such that the image by $R$ of the $L$-ball in $H^{\infty}(0)$ is $\varepsilon$-dense in the unit ball in $\left.H^{\infty}(V)\right|_{s}$. (See for example Lemma 1.4. in [11].) Choose $f$ in the unit ball of $\left.H^{\infty}(V)\right|_{S}$. By (ii) and the open mapping theorem there is a constant $c_{1}$ independent of $f$, and $f_{1} \in H^{\infty}(U)$ such that $\left.f_{1}\right|_{s}=f$ and $\left\|f_{1}\right\|_{U} \leq c_{1}$. By Lemma 3.2 in [11] we can choose 0 such that for each $g \in H^{\infty}(U)$ there exists $g_{1} \in H^{\infty}(0)$ such that
(1) $\left\|g_{1}\right\|_{0} \leq c_{2}\|g\|_{U}$
(2) $\left\|g-g_{1}\right\|_{V} \leq\left(2 c_{1}\right)^{-1}\|g\|_{U}$

where $c_{2}$ is independent of $g$. (That we actually can apply Lemma 3.2 in [11] in this situation follows from well known estimates of analytic capacity. See for example the proof of Theorem 7.4 on page 213 in [6]). If we replace $g$ by $\left(f_{1}\right)$ in (1) and (2), we see that with $\epsilon=1 / 2$ and $L=c_{1} c_{2}$, Lemma 1.4 in [11] can be applied.

To see that (i) $\Rightarrow$ (iii) we first observe that the restriction map $R$ 
defined above is not one-to-one. If it was, $\|f\|_{0}$ and $\|f\|_{U}$ would be equivalent norms on $H^{\infty}(0)$ by (i) and the open mapping theorem, and that is absurd. Hence there is some function $h \in H^{\infty}(0)$ which is zero on $S$ but not identically zero in $U$. Choose $m \in M$ such that $\Pi(m)=z_{0} \in \bar{U} \backslash \bar{S}$. Since $h$ is analytic near $z_{0}$ we can write $h-h\left(z_{0}\right)=\left(z-z_{0}\right) h_{1}$ where $h_{1} \in H^{\infty}(0)$. If we apply $m$ on the right side we get zero and therefore $m(h)=h\left(z_{0}\right)$. Since we clearly can assume $h\left(z_{0}\right) \neq 0$ we have proved that $m \notin S$ and (iii) follows.

It remains to prove that (iii) $\Rightarrow$ (ii) and here we apply Carleson's lemma. (See [2] or on page 203 in [4].) Let $\varphi: U \rightarrow D$ be a conformal map and put $S_{1}=\varphi(s)$. By (iii) $S_{1}$ must be countable and we let $B$ denote the Blaschke product corresponding to $S_{1}$. For definition and basic properties of Blaschke products we refer to [4] page 20 or [9] page 66. From these properties it is easy to see that $V_{1}=\left\{z:|B(z)|<2^{-1}\right\}$ satisfies $\bar{V}_{1} \backslash\left(\bar{S}_{1} \backslash S_{1}\right) \subset D$ and Carleson's lemma ([4] page 203) combined with a simple normal family argument, gives that $\left.H^{\infty}(D)\right|_{S_{1}}=\left.H^{\infty}\left(V_{1}\right)\right|_{S_{1}}$. If we define $V=\varphi^{-1}\left(V_{1}\right)$, it only remains to prove that $\bar{V} \backslash(\bar{S} \backslash S) \subset U$. Put $g=$ $B \circ \varphi$. Choose an arbitrary point $z_{0} \in(\partial U) \backslash \bar{S}$. If we can show that $\left|g\left(w_{n}\right)\right|$ $\rightarrow 1$ whenever $\left\{w_{n}\right\}_{n=i}^{\infty} \subset U$ converges to $z_{0}$, the proof will be complete.

Let $\left\{z_{n}\right\}$ be an arbitrary sequence in $U$ converging to $z_{0}$. We denote by $J$ the ideal of all $h \in H^{\infty}(U)$ satisfying $\lim h\left(z_{n}\right)=0$. We want to show that $g \notin J$. Let $m$ denote some maximal ideal containing $J$. Since $J$ contains the translation $z \rightarrow z-z_{0}$ we get that $\Pi(m)=z_{0}$. If $g \in J$ and $f \in H^{\infty}(U)$ vanishes on $S$, we can write $f=g f_{1}$, with $f_{1} \in H^{\infty}(U)$. (see Thm. 2.8 on page 24 in [4]) and hence we get $m(f)=m(g) m\left(f_{1}\right)=0$. This implies $m \in$ $\tilde{S}$ which is impossible by (iii) and since $\Pi(m)=z_{0}$. We can therefore

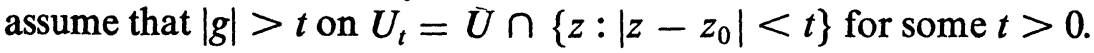

The proof is completed using some well known facts about $H^{\infty}(U)$ which we shall not prove. But references will be given below. We fix a point $w \in U$ and let $\lambda$ denote the harmonic measure on $\partial U$ which represents $w$. There is a (unique) function $g^{*} \in L^{\infty}(\lambda)$ whose harmonic extension to $U$ equals $g$. (See for example [15] page 26.) We now claim:

(a) Since $|B|=1$ a.e. on $\partial D$ with respect to linear measure, $\left|g^{*}\right|=1$ a.e. with respect to $\lambda$.

(b) Define $g_{1}$ on $\partial U_{t}$ by $g_{1}=g$ on $\left(\partial U_{t}\right) \cap U$ and $g_{1}=g^{*}$ on $\left(\partial U_{t}\right) \backslash U$. Then the harmonic extension of $g_{1}$ to $U_{t}$ equals the restriction $g_{2}$ of $g$ to $U_{t}$. We can also assume that $\left|g_{1}\right|=1$ on $\left(\partial U_{t}\right) \backslash U$.

Since $|g|>t$ on $U_{t}$ we have from Jensen's inequality ([6] page 33-34) and $(b)$ that the harmonic extension of $\log \left|g_{1}\right|$ to $U_{t}$ equals $\log \left|g_{2}\right|=\log |g|$. But if $\left\{w_{n}\right\} \subset U$ converges to $z_{0}$, we get that $\log \left|g_{2}\left(w_{n}\right)\right| \rightarrow 0$ since $z_{0}$ is regular for the Dirichlet problem for $U_{t}$. Since $g_{2}=g$ in $U_{t}$ this completes 
the proof that (iii) $\Rightarrow$ (ii). The claims (a) and (b) above are easy to justify using well known theory about harmonic measure and algebras of analytic functions. A convenient reference is the introductory part of [7]. (See in particular Lemma 2.2 and Lemma 4.4 in [7].)

We shall now prove that $\left(^{*}\right)$ holds for all the Hardy spaces $H^{p}(D), 0<$ $p \leq \infty$ and with $0=C \backslash(\bar{S} \backslash S)$. We first prove a general result which may be of independent interest.

THEOREM 2. Let $A$ be a Banach space of functions on $D$ with norm $N(\cdot)$. Assume $A$ contains the polynomials in $z$ and there exists constants $M_{n}$, $n=1,2, \ldots$ such that:

$$
N\left(\left.p\right|_{D}\right) \leq M_{n} \sup \left\{|p(z)|:|z| \leq 1+n^{-1}\right\} \text { for } n=1,2, \ldots
$$

if $p$ is a polynomial. For each $z \in D$ assume the map $f \rightarrow f(z)$ is continuous on $A$.

Let $S \subset D$ and assume there exists an open set $0 \supset \bar{D} \backslash(\bar{S} \backslash D)$ such that each $\left.g \in A\right|_{S}$ extends to a function $f$ analytic in 0 such that $f_{D} \in A$. Then such a function exists which even extends to be analytic in $\mathbf{C} \backslash(\bar{S} \backslash D)$.

Remarks. Note that (1) implies $f_{D} \in A$ whenever $f$ is analytic in a neighbourhood of $\bar{D}$ and that we have estimates like (1) also for such functions.

Proof of Theorem 2. Denote by $A_{1}$ all analytic functions in 0 whose restriction to $D$ are in $A$. We topologize $A_{1}$ by saying that a sequence $\left\{f_{n}\right\}$ $\in A_{1}$ converges to $f \in A_{1}$ if and only if $N\left(\left.\left(f_{n}-f\right)\right|_{D}\right) \rightarrow 0$ and $\left\|f-f_{n}\right\|_{K} \rightarrow$ 0 if $K$ is a compact subset of 0 .

With this topology $A_{1}$ is a Frechet space and we can apply the open mapping theorem to the restriction map $\left.A_{1} \rightarrow A\right|_{S}$ where $\left.A\right|_{S}$ has the quotient norm induced from $A .\left.A\right|_{S}$ is then a Banach space since the set of functions in $A$ vanishing on $S$ must be closed by hypothesis. Choose an open set $0_{1} \supset \bar{D} \backslash(\bar{S} \backslash D)$ such that $\sigma_{1} \backslash \bar{D} \subset 0$. By the open mapping theorem there exists a constant $M$ and constants $M_{K}$ for each compact subset $K$ of $\overline{0}_{1} \backslash(\bar{S} \backslash D)$ such that each $g$ in the unit ball of $\left.A\right|_{s}$ extends to $h$ $\in A_{1}$ such that

(i) $N\left(\left.h\right|_{D}\right) \leq M$

(ii) $|h| \leq M_{K}$ on $K$ if $K \subset \overline{0} \backslash(\bar{S} \backslash D)$ is compact

Now redefine $h$ by setting $h \equiv 0$ in $C \backslash \bar{\sigma}_{1}$. When we in the rest of the proof of Theorem 2 claim that a property holds independent of $h$, we shall mean 
that this property holds for all $h \in A_{1}$ satisfying (i) and (ii) as above and extended to $\mathbf{C}$ as above.

We can and shall assume $0_{1}$ has the following property:

(2) $C \backslash \bar{\sigma}_{1}$ is connected and there exists a constant $L$ such that each $z \in$ $C \backslash \overline{0}_{1}$ can be connected to a point in $\bar{S}$ by an $\operatorname{arc} \gamma_{z} \subset C \backslash \overline{0}_{1}$ such that (length of $\left.\gamma_{z}\right) \leq L$ dist $(z, \bar{S} \backslash D)$.

With the notation as above the following lemma completes the proof of Theorem 2:

LEMMA 1. Given $t>0$ there exists constants $C_{K}$ for each compact subset $K$ of $C \backslash(\bar{S} \backslash S)$ such that for each function $h$ as above we can find $h_{1}$ analytic in $\mathbf{C} \backslash(\bar{S} \backslash D)$ such that $\left.h_{1}\right|_{D} \in A$ with the following properties:

(a) $N\left(\left.\left(h-h_{1}\right)\right|_{D}\right) \leq t$

(b) $\left|h_{1}\right| \leq \mathbf{C}_{K}$ on each compact subset $K$ of $\mathbf{C} \backslash(\bar{S} \backslash D)$.

Indeed if Lemma 1 is proved, Theorem 2 follows by the same iteration argument as in the proof of Lemma 1.4 in [11].

The first part of the proof of Lemma 1 is very similar to the proof of Lemma 3.2 in [11], but for completeness we give most of the details.

Let $\left\{K_{n}\right\}_{n=1}^{\infty}$ be compact sets, $\left\{V_{n}\right\}_{n=1}^{\infty}$ open sets with the following properties:

(i) $K_{n} \subset V_{n}, n=1,2, \ldots$

(ii) $\bar{V}_{n} \cap \bar{D}=\phi, n=1,2, \ldots$

(iii) $\bar{V}_{n} \cap \bar{V}_{m}=\varnothing$ if $|n-m|>1$

(iv) $\left(\mathrm{\partial O}_{1}\right) \backslash \bar{D}=\bigcup_{n} K_{n}$

(v) For each compact set $F \subset C \backslash(\bar{S} \backslash D), F \cap \bar{V}_{n}=\phi$ if $n$ is sufficiently large.

Fix $n$ : Put $K=K_{n}, V=V_{n}$ and let $\varepsilon=\varepsilon_{n}$ be a positive number. Let $\delta>0$ be given. Then cover $C$ by open discs $\Delta_{k}=\Delta\left(z_{k}, \delta\right)$ (of radius $\delta$ and centered at $z_{k}$ ) and choose continuously differentiable functions $\phi_{k}$ (supported at $\Delta_{k}$ ) as in the scheme for approximation described on page 210 in [6].

Let $T_{\phi_{k}}$ be the integral operator on $L^{\infty}(d x d y)$ defined by

$$
\begin{aligned}
& T_{\varphi_{k}}(f)(w)=\frac{1}{\pi} \iint \frac{f(w)-f(z)}{w-z} \frac{\partial \varphi}{\partial z} d x d y \\
& =f(w) \varphi_{k}(w)+\frac{1}{\pi} \iint \frac{f(z)}{z-w} \frac{\partial \varphi_{k}}{\partial z} d x d y
\end{aligned}
$$


We mention that $T_{\phi_{k}}(f)$ is analytic outside the support of $\phi_{k}$ and wherever $f$ is and that $T_{\phi_{k}}(f)$ is continuous wherever $f$ is. Also $f-T_{\phi_{k}}(f)$ is analytic in the interior of the set where $\phi_{k}$ attains the value 1. (See on page 28-29 in [6] for more details.)

Put $G_{k}=T_{\phi_{k}}^{\prime}(h)$ where $h$ is as above. We are only interested in those $k$ for which $\bar{\Delta}_{k} \cap K \neq \varnothing$. Assume this happens if and only if $1 \leq k \leq N$.

Then $h-\Sigma_{1}^{N} G_{k}$ is analytic near $K$ since $\Sigma_{1}^{N} G_{k}=T_{\left(\Sigma_{1}^{N} \phi_{k}\right)}(h)$ and $\Sigma_{1}^{N} \varphi_{k} \equiv$ 1 in a neighborhood of $K$. We can assume $\delta>0$ is so small that $\left\{z:\left|z-z_{k}\right|\right.$ $\leq 2 \delta\} \subset V$ for $1 \leq k \leq N$.

Now there exist functions $H_{k}, k=1, \ldots, N$ analytic outside a compact subset of $D_{k}=\left\{w:\left|w-z_{k}\right|<2 \delta\right\} \backslash \overline{0}_{1}$ such that $G_{k}-H_{k}$ has a triple zero in the Taylor expansion at infinity, and in our situation (since $C \backslash \overline{0}_{1}$ is connected) we obtain $\left\|H_{k}\right\|<C_{1}\|h\|_{V}$ where $C_{1}$ is an absolute constant. (See [6], Theorem 7.4 on page 213 and the proof of it). The important fact is that $C_{1}$ is independent of $h$.

We now list the facts which will be needed to prove Lemma 1.

(a) One can choose $\delta$ depending only on $\varepsilon$ and dist $(K, \mathbf{C} \backslash V)$ so small that the function $f=\Sigma_{1}^{N}\left(G_{k}-H_{k}\right)$ satisfies

$$
\|f\|_{\mathbf{C} \backslash V}<\varepsilon\|h\|_{V}
$$

and we also have $\|f\|_{\infty} \leq C_{2}\|h\|_{V}$ where $C_{2}$ is independent of $h .\left(\|f\|_{\infty}\right.$ denotes ess.sup. of $|f|$ with respect to plane measure.)

(b) The functions $H_{k}$ can be written as

$$
H_{k}=\alpha_{k}(h) F_{k, 1}+\beta_{k}(h) F_{k, 2}
$$

where $F_{k, 1}$ and $F_{k, 2}$ both are analytic outside a compact subset of $D_{k}$, they are independent of $h$ and $\left\|F_{k, 1}\right\|_{\infty}+\left\|F_{k, 2}\right\|_{\infty} \leq 20$.

Here $\alpha_{k}(h)$ and $\beta_{k}(h)$ are complex numbers depending linearly on $h$ and we have

$$
\left|\alpha_{k}(h)\right|+\left|\beta_{k}(h)\right| \leq C_{3}\|h\|_{V}
$$

where $C_{3}$ is independent of $h$. (See the proof of Theorem 3.1 in [11] for more details about this.)

The functions $F_{k, 1}$ and $F_{k, 2}$ can now be approximated as well as we please in $\mathbf{C} \backslash D_{k}$ by rational functions $R_{k, 1}$ and $R_{k, 2}$ with their poles in $D_{k} k$ $=1,2, \ldots, N$ so that if we define

$$
f^{*}=\sum_{k=1}^{N} G_{k}-\alpha_{k}(h) R_{k, 1}+\beta_{k}(h) R_{k, 2}
$$


then we have

$$
\left\|f^{*}\right\|_{\mathbf{C} \backslash V}<\varepsilon\|h\|_{V}<\varepsilon C_{V}
$$

where $C_{V}$ is a constant depending only on $V$. The existence of $C_{V}$ comes from property (ii) of $h$ listed above, and since $\bar{V} \cap \bar{D}=\varnothing$.

Note that from the remark following Theorem 2 there exists a constant $C_{V}^{\prime}$ also depending only on $V$ such that from (5) we have

$$
N\left(\left.f^{*}\right|_{D}\right) \leq \varepsilon C_{V}^{\prime}\|h\|_{V}<\varepsilon C_{V} C_{V}^{\prime}
$$

Let now $n$ vary and carry out this construction with $V=V_{2 n-1}$ and $\varepsilon=$ $\varepsilon_{n}, n=1,2, \ldots$. In this way we obtain functions $f_{n}{ }^{*}, n=1,2, \ldots$ with the same properties as $f^{*}$ has above. We can choose $\varepsilon_{n}$ independent of $h$ such that

$$
\left\|f_{n}\right\|_{\mathbf{C} \backslash V_{2 n-1}}+N\left(\left.f_{n}^{*}\right|_{D}\right)<t\left(2^{-2}\right) 2^{-n}
$$

where $t$ is the number in Lemma 1 .

Now define $h^{\prime}=h-\Sigma_{n} f_{n}{ }^{*}$. By (6) and property (iii) of $\left\{V_{n}\right\}, h^{\prime}$ has the following property

$$
\left.h^{\prime}\right|_{D} \in A \text { and } N\left(\left.\left(h^{\prime}-h\right)\right|_{D}\right)<t \cdot 2^{-2}
$$

We now wish to repeat this construction with $h$ replaced by $h^{\prime}$ and $V_{2 n-1}$ by $V_{2 n}, n=1,2, \ldots$. We have to be a bit careful because $h^{\prime}$ can be unbounded in $V_{2 n}$ for some $n$. But for $n=1,2, \ldots$ it is easy to see that we can find open sets $W_{n} \subset V_{2 n}$ such that $K_{2 n} \subset W_{n}$ and such that none of the rational functions $R_{k, 1}$ or $R_{k, 2}$ used in the definition of $f_{n}{ }^{*}, n=1,2, \ldots$ has poles in $W_{n}$. But then it follows that there exists constants $E_{n}, n=1,2, \ldots$ independent of $h$ and $h^{\prime}$ such that

$$
\left\|h^{\prime}\right\|_{W_{n}} \leq E_{n} \text { for } n=1,2, \ldots
$$

We can now repeat the above construction with $h$ replaced by $h^{\prime}$ and $V_{2 n-1}$ replaced by $W_{n}$ for $n=1,2, \ldots$. We obtain functions $g_{n}{ }^{*}$ analytic in C $\backslash W_{n}$ in the same way as we obtained $f_{n}{ }^{*}$.

Define $h^{*}=h-\Sigma_{n} f_{n}{ }^{*}-\Sigma_{n} g_{n}{ }^{*}$. In the same way as we obtained (7) we get

$$
\left.h^{*}\right|_{D} \in A \text { and } N\left(\left.\left(h^{*}-h\right)\right|_{D}\right)<t \cdot 2^{-1} \text {. }
$$


From the properties of the $T_{\varphi}$-operator mentioned above one can also deduce that $h^{*}$ is analytic in $C \backslash(\bar{S} \backslash D)$ except for the poles of the rational functions $R_{k, 1}$ and $R_{k, 2}$ corresponding to each $f_{n}{ }^{*}$ and each $g_{n}{ }^{*}$.

Let now $K$ be a compact subset of $C \backslash(\bar{S} \backslash D)$ and let $\Sigma_{n}^{\prime}$ denote summation over those $n$ for which $\bar{W}_{n} \cap K=\phi$ and $V_{2 n-1} \cap K=\phi$. It is easy to see that there exists a constant $E_{K}$ depending on $K$ but not on $h$ such that

$$
\left\|h-\sum_{n}^{\prime}\left(f_{n}^{*}+g_{n}^{*}\right)\right\|_{K} \leq E_{K}
$$

We conclude that our function $h^{*}$ satisfies almost Lemma 1 . We get rid of the rational functions $R_{k, 1}$ and $R_{k, 2}$ by the following lemma

LEMMA 2. Suppose $\eta>0$ is given. Let $p$ be a rational function with poles only at the points $z_{1}, \ldots, z_{m}$ in $\mathbf{C} \backslash \bar{\sigma}_{1}$. Then there exists a function $s$ analytic in $\mathbf{C} \backslash(\bar{S} \backslash D)$ and an open set $W \subset \mathbf{C} \backslash(\bar{S} \backslash D)$ such that

(i) $\left.s\right|_{D} \in A$ and $\|s-p\|_{C \backslash W}+N\left(\left.(s-p)\right|_{D}\right)<\eta$

(ii) $\operatorname{dist}(z, \bar{S} \backslash D)<2 L \max _{1 \leq k \leq m} \operatorname{dist}\left(z_{k}, \bar{S} \backslash D\right)$ for

each $z \in W$, where $L$ is as in condition (II) mentioned above.

Proof. It is clearly sufficient to prove this lemma when $m=1$. We choose a polygonal arc $\gamma=\gamma_{z 1}$ as in condition (2).

Divide $\gamma$ into subarcs $\gamma_{k}$ with endpoints $z_{k}$ and $z_{k+1}, k=1,2, \ldots$ such that $z_{k+1}$ is the only common point of $\gamma_{k}$ and $\gamma_{k+1}$ for each $k$.

Choose connected open sets $U_{k} \supset \bar{\gamma}_{n}$ for $k=1,2, \ldots$ and rational functions $p_{k}, k=1,2, \ldots$ (with $p=p_{1}$ ) with poles only at $z_{k}$ such that

$$
\left\|p_{k+1}-p_{k}\right\|_{\mathbf{C} \backslash U_{k}}+N\left(\left.\left(p_{k+1}-p_{k}\right)\right|_{D}\right)<\eta 2^{-k}
$$

$k=1,2, \ldots$. Since each $U_{k}$ is connected and since we can assume $\bar{U}_{k} \cap \bar{D}$ $=\phi$ this is easy to obtain. We can also assume $\bar{U}_{k} \cap K=\phi$ if $k$ is sufficiently large and $K$ is a given compact subset of $\mathbf{C} \backslash(\bar{S} \backslash D)$. Since the length of $\gamma$ is less than $L$ dist $\left(z_{1}, \bar{S} \backslash D\right)$ it is easy to see that we can choose $U_{k} k=1,2, \ldots$ such that $W=\cup_{k} U$ satisfies (ii). But then $p_{k}$ converges to a function $s$ which satisfies our requirements.

It is now relatively easy to complete the proof of Lemma 1. Each of the functions $f_{n}{ }^{*}$ and $g_{n}{ }^{*}$ can be written as finite sums of the form (4). (For $g_{n}{ }^{*}$ one must replace $h$ by $h^{\prime}$ in (4).) The rational functions $R_{k, 1}$ and $R_{k, 2}$ are independent of $h$ and we have also bounds for the constants $\alpha_{k}(h)$ and 
$\beta_{k}(h)$ which are independent of $h$. (See [3] and the remark following (5).) If one applies Lemma 2 with care and approximate the functions $R_{k, 1}$ and $R_{k, 2}$ by functions $S_{k, 1}$ and $S_{k, 2}$ analytic in $\mathbf{C} \backslash(\bar{S} \backslash D)$ using that lemma, we get "new" functions $f_{n}{ }^{* *}$ and $g_{n}{ }^{* *}$ by replacing $R_{k, 1}$ and $R_{k, 2}$ by $S_{k, 1}$ and $S_{k, 2}$ in the expressions of the form (4) for $f_{n}{ }^{*}$ and $g_{n}{ }^{*}$. Define then

$$
: h_{1}=h-\sum_{n}\left(f_{n}^{* *}+g_{n}{ }^{* *}\right) \text {. }
$$

Note from property (v) of $\left\{V_{n}\right\}$ that if $U \supset(\bar{S} \backslash D)$ is open then there exists a number $N$ such that the poles of the rational functions $R_{k, 1}$ and $R_{k, 2}$ corresponding to $f_{n}{ }^{*}$ and $g_{n}{ }^{*}$, must be contained in $U$ if $n \geq N$. From this fact and (ii) in Lemma 2 it is easy to see that the series (11) will converge uniformly on compact subsets of $\mathbf{C} \backslash(\bar{S} \backslash D)$. From (9) and (10) it follows that $h_{1}$ will satisfy Lemma 1 if Lemma 2 is applied carefully. We don't want to go into further details about this.

Using Theorem 2 we shall now prove:

TheOREM 3. Assume $S=\left\{z_{n}\right\} \subset D$ satisfies $\Sigma_{n}\left(1-\left|z_{n}\right|\right)<\infty$. If 0 $<p \leq \infty$ and $\left.f \in H^{p}(D)\right|_{s}$, there exists $g$ analytic in $\mathbf{C} \backslash(\bar{S} \backslash D)$ such that $\left.g\right|_{D} \in H^{p}(D)$ and $g=$ fon $S$.

Proof. Assume first Theorem 3 is proved for $1<p \leq \infty$. If $g \in$ $H^{p}(D)$ has no zeros in $D, g$ has a $k^{\prime}$ th root for some integer $k$ such that $k p>$ 1. By assumption we can find $f$ in $H^{k p}(D)$ which interpolates this $k^{\prime}$ th root on $S$ and extends to be analytic in $\mathbf{C} \backslash(\bar{S} \backslash D)$. But $\left.f^{k}\right|_{D} \in H^{p}(D)$ and interpolates $g$ on $S$. Since an arbitrary function in $H^{P}(D)$ can be written as the sum of two functions in $H^{p}(D)$ with no zeros in $D$, ([4], page 79) Theorem 3 will be true for all $p>0$ if it holds for $1<p \leq \infty$. By Theorem 2 we need only prove the following for $1<p \leq \infty$ :

(***) There exists an open set $0 \supset \bar{D} \backslash(\bar{S} \backslash D)$ such that each $f$ in $\left.H^{P}(D)\right|_{s}$ extends to a function $h$ analytic in 0 such that $\left.h\right|_{D} \in H^{P}(D)$.

If $p=\infty$ this is just the result $\left({ }^{*}\right)$ proved by Detraz [3]. Her methods seem to work also if $1<p<\infty$, but some additional results from the theory of $H^{p}$-spaces are needed. We give here a different proof for $1<p<$ $\infty$.

We first need an approximation result for $H^{p}(D)$ similar to Lemma 3.2 in [11]. If $f \in H^{p}(D), 1<p<\infty,\|f\|_{P}$ denotes its norm in $H^{p}(D)$. 
Lemma 3. Assume $1<p<\infty$. There exists a constant $C_{p}$ depending only on $p$ such that for each $\varepsilon>0$ and each relatively closed set $F \subset D$ we can find an open set $0 \supset \bar{D} \backslash(\bar{F} \backslash D)$ with the following properties:

Given $f \in H^{p}$ there exists $g$ analytic in 0 such that $\left.g\right|_{D} \in H^{p}$ and

(a) $\sup \{|f(z)-g(z)|, z \in F\}<\varepsilon\|f\|_{p}$,

(b) $\left\|\left.g\right|_{D}\right\|_{P} \leq C_{p}\|f\|_{p}$

(c) for each set $K \subset 0$ with $\operatorname{dist}(K, \bar{F} \backslash D)>0$ we have $\sup \{|g(z)|, z \in K\}$ $<C_{K}\|f\|_{p}$ where $C_{K}$ is independent off.

To prove Lemma 3 it is convenient first to establish the following:

LEMma 4. Assume $1<p<\infty$ and $f \in H^{p}(D)$. If $\varphi$ is a measurable function on the unit circle $T$ we define

$$
S \varphi f(z)=\frac{1}{2 \pi} \int_{-\pi}^{\pi} \frac{e^{i \theta}+z}{e^{i \theta}-z} \operatorname{Re} f\left(e^{i \theta}\right) \varphi\left(e^{i \theta}\right) d \theta
$$

if $z$ is outside the closed support supp $\varphi$ of $\varphi$. Assume $0 \leq \varphi \leq 1$.

If $K \subset \mathbf{C}$ and $\operatorname{dist}(K, \operatorname{supp} \varphi)>0$ we have $\sup \{|S \varphi f(z)|, z \in K\} \leq M_{p}$ $\operatorname{dist}(K, \operatorname{supp} \varphi)^{-1}\|f\|_{P}$ where $M_{p}$ is a constant depending only on $p$.

Proof of Lemma 4. Since we on $T$ have $\operatorname{Re} S \varphi f=\varphi \operatorname{Re} f$, Lemma 4 is an immediate consequence of a well known theorem on M. Riesz ([4], Thm. 4.1, page 54) and $\mathrm{H} \varnothing$ lder's inequality.

Proof of Lemma 3. We choose open plane sets $V_{j}, j=1,2, \ldots$ satisfying:

(i) $T \backslash \bar{F} \subset \cup_{1}^{\infty} V_{j}$,

(ii) $\bar{V}_{j} \cap \bar{V}_{i}=\varnothing$ if $|i-j|>1$,

(iii) $\bar{F} \cap \bar{V}_{j}=\phi$ for $j=1,2, \ldots$,

and (iv) if $K \subset \mathbf{C} \backslash(\bar{F} \backslash D)$ is compact there are at most finitely many $j$ such that $K \cap \bar{V}_{j} \neq \varnothing$.

We also choose functions $\varphi_{j} \in C^{\infty}(T)$ such that $0 \leq \varphi_{j} \leq 1$, supp $\varphi_{j} \subset$ $V_{j}$ and $\Sigma_{1}^{\infty} \varphi_{j}=1$ on $T \backslash \bar{F}$.

Given $f \in H^{p}$ we put $f_{j}=S \varphi_{j}(f), j=1,2, \ldots$ where $S \varphi_{j}(f)$ is defined as in Lemma 4. From the arguments used to prove Lemma 4 it is easy to see that we can choose numbers $r_{j} \in(0,1), j=1,2, \ldots$ independent of $f$ such that the functions $h_{j}: z \rightarrow f\left(r_{j} z\right)$ satisfies

(1): $\sup \left\{\left|f_{j}(z)-h_{j}(z)\right|: z \in \mathbf{C} \backslash V_{j}\right\}<\varepsilon 2^{-j}\|f\|_{p}$ for $j=1,2, \ldots$ 
Define $g=f-\sum_{j=1}^{\infty}\left(f_{j}-h_{j}\right)$. By (1), (a) in Lemma 3 is valid. Consider a point $w \in T \backslash \bar{F}$. There exists by (i) and (ii) a number $k$ and a $\operatorname{disc} \Delta(w)$ centered at $w$ such that $\overline{\Delta(w)} \cap \bar{V}_{j}=\phi$ if $j \notin\{k, k+1\}$.

Write

$$
\begin{gathered}
g=\left(f-f_{k}-f_{k+1}\right)+\left(h_{k}+h_{k+1}\right)+\left(\sum_{j=k, k+1}\left(h_{j}-f_{j}\right)\right) \\
=F_{1}+F_{2}+F_{3}
\end{gathered}
$$

say. Here $F_{1}$ can be written as $S \varphi f$ where $\varphi=1-\varphi_{k}-\varphi_{k+1}$ must have compact support disjoint from $\overline{\Delta(w)}$. So $F_{1}$ is analytic in $\Delta(w)$ and by Lemma $4 \sup \left\{\left|F_{1}(z)\right|, z \in \Delta(w)\right\} \leq C_{w}\|f\|_{p}$ where $C_{w}$ depends only on $\operatorname{dist}(\operatorname{supp} \varphi, \Delta(w))$. Clearly also $F_{3}$ is analytic in $\Delta(w)$ and by (1) $\sup \left\{\left|F_{3}(z)\right|\right.$, $z \in \Delta(w)\} \leq \varepsilon\|f\|_{p}$. Put $t=\max \left\{r_{k}, r_{k+1}\right\}$. Then $F_{2}$ is analytic in $\{z:|z|<$ $\left.t^{-1}\right\}$.

Define $D(w)=\Delta(w) \cap\left\{z:|z|<\left(1+t^{-1}\right) 2^{-1}\right\}$. Again by Lemma 4 we obtain $\sup \left\{\left|F_{2}(z)\right|, z \in D(w)\right\} \leq C_{w}^{1}\|f\|_{p}$ where $C_{w}^{1}$ depends only on $t$.

Let $D_{j}=D\left(w_{j}\right), j=1,2, \ldots$ denote a locally finite covering of $T \backslash \bar{F}$ by such sets. We define $0=D \cup\left(\cup_{j} D_{j}\right)$.

To verify (c) in Lemma 3 let $K \subset 0$ have positive distance from $\bar{F} \backslash D$. Then we can write $K=K_{1} \cup K_{2}$ where $\bar{K}_{1}$ is a compact subset of $D$ and $K_{2}$ $\subset \cup_{1}^{N} D_{j}$ for some number $N$. It is easy to verify (c) on $K_{1}$ and $K_{2}$ separately.

It remains to verify (b). Consider the point $w \in T \backslash \bar{F}$ again. We have $|\operatorname{Re} g(w)| \leq \varepsilon\|f\|_{P}+\left|h_{k}(w)\right|+\left|h_{k+1}(w)\right|$

$$
\begin{aligned}
& \leq \varepsilon\|f\|_{p}+\sup _{0<r<1}\left|f_{k}(r w)\right|+\sup _{0<r<1}\left|f_{k+1}(r w)\right| \\
& \leq \varepsilon\|f\|_{p}+2 \sup _{0<r<1} u(r w)=\varepsilon\|f\|_{p}+\eta(w)
\end{aligned}
$$

where $u$ is the harmonic extension to $D$ of $|f|$.

Finally let $w \in \bar{F} \backslash D$. We can clearly assume $\bar{V}_{j} \cap r z=\phi$ for all $j$, all $z \in \bar{F} \backslash D$ and all $r \in(0,1)$. But this implies

$$
|\operatorname{Re} g(w)| \leq \varepsilon|| f \|_{p}+|\operatorname{Re} f(w)|
$$

By a theorem of Hardy and Littlewood $\|\eta\|_{p} \leq A_{p}\|f\|_{p}$ where $A_{p}$ depends only on $p$. But then $\|\operatorname{Re} g\|_{p} \leq K_{p}\|f\|_{p}$ where $K_{p}$ depends only $p$ and by the 
theorem of M. Riesz used in the proof of Lemma 4, (b) follows. The Hardy-Littlewood result is in [4, Thm. 1.9, p. 12].

To complete the proof of the above claim about $H^{p}(D)$ we need a result similar to (**) for $H^{p}(D)$ when $1<p<\infty$.

We need some notation. Let $\Gamma$ be a simple closed rectifiable curve and denote by $0_{\Gamma}$ the bounded component of $C \backslash \Gamma$. Let $\mu$ denote the arc length measure associated with $\Gamma$. So $\mu(E)$ is the length of $E \cap \Gamma$ for each Borel set $E$. If $1<p<\infty, H^{P}(\Gamma)$ denotes the closure in $L^{P}(\mu)$ of the polynomials in $z$. The functions in $H^{P}(\Gamma)$ can be extended to analytic functions in $0_{\Gamma}$ by Cauchy's integral formula and we shall assume them extended in this way.

Lemma 5. Let $S=\left\{z_{n}\right\} \subset D$ satisfy $\Sigma_{n}\left(1-\left|z_{n}\right|\right)<\infty$. Then there exists a contour $\Gamma$ such that $\overline{0}_{\Gamma} \backslash(\bar{S} \backslash S) \subset D, 0_{\Gamma} \supset S$ and $\left.H^{p}(\Gamma)\right|_{S}=$ $\left.H^{p}(D)\right|_{s}$ for $1 \leq p \leq \infty$.

Proof. This result is essentially contained in Carleson's lemma ([4], page 203) and the proof we give has all its basic ideas contained in the proof of Carleson's lemma. Let $B(z)$ be the Blaschke product corresponding to $S$ and let $B_{N}$ consist of the first $N$ factors in the product defining $B$. Let

$$
S_{1}=\left\{z \in D:|B(z)| \leq 2^{-1}\right\} \text {. Then } \bar{S}_{1} \backslash S_{1}=\bar{S} \backslash S \text {. }
$$

Let now $T \backslash \bar{S}$ consist of the disjoint $\operatorname{arcs} J_{n}, n=1,2, \ldots$. For each $n$ we choose a simple arc $I_{n} \subset D \backslash\{0\}$ with endpoints equal to the endpoints of $J_{n}$ and with the radial projection onto $T$ equal to $J_{n}$. We wish to do this in such a way that the arclength measure associated with $U_{n} I_{n}$ is a Carleson measure. (See [4] page 157 for definition.) We indicate one way of doing this. Assume for simplicity that $J_{n}=\left\{e^{i \theta}:-a<\theta<a\right\}$ for some $a \in(0$, $\pi)$. Let $\left\{a_{k}\right\} \subset(0, a)$ and $\left\{r_{k}\right\} \subset(1-a / \pi, 1)$ be monotonic sequences converging to $a$ and 1 respectively. Assume that $R_{k}=\left\{r e^{i \theta}:|\theta|<a_{k}, r_{k}<\right.$ $r<1\}$ is disjoint from $S_{1}$ and $1-r_{k}<a-a_{k}$ for all $k$. Define $I_{n}=D \cap \partial$ $\left(\cup_{k} R_{k}\right)$. It is easy to verify that $\left\{I_{n}\right\}$ has all the required properties.

Define $\Gamma=(\bar{S} \backslash S) \cup\left(\cup_{n} I_{n}\right)$. Fix an integer $N$ and choose $f \in H^{p}(\Gamma)$. As in [4] page 204 and 139-140, we get that the function $g_{N}$ in $H^{p}(D)$ of minimal norm which interpolates $f$ on $\left\{z_{1}, \ldots, z_{N}\right\}$ must satisfy

$$
\left\|g_{N}\right\|_{p} \leq\left|(2 \pi i)^{-1} \int_{\Gamma} h(z) f(z)\left(B_{N}(z)\right)^{-1} d z\right|
$$

for some $h \in H^{q}(D)$ of norm one and where $p^{-1}+q^{-1}=1$. Since $\left|B_{N}\right| \geq$ $|B| \geq 2^{-1}$ on $\Gamma$ and the arc length measure associated with $\Gamma \cap D$ is a Carleson measure we get by using $\mathrm{H} \phi$ lder's inequality that 


$$
\left\|g_{N}\right\|_{P} \leq C_{1}\|f\|_{L} p_{(\mu)} \text { where } C_{1}
$$

depends only on $\Gamma$. (See Theorem 9.3 on page 157 in [4].) A subsequence of $\left\{g_{N}\right\}$ converges uniformly on compact subsets of $D$ to a function $g$ which satisfies Lemma 5.

The result $\left({ }^{* * *}\right)$ for $1<p<\infty$ is now easy to prove. It follows from Lemma 3 and Lemma 5 in the same way as we proved (ii) $\Rightarrow$ (i) in Theorem 1 .

We finally apply Theorem 2 to a result of Vinogradov [12]. Again let $S$ $=\left\{z_{n}\right\} \subset D$. We shall need the following condition on $S$ :

$$
\inf _{k} \prod_{\substack{n=1 \\ n \neq k}}\left|\frac{z_{n}-z_{k}}{1-\bar{z}_{k} z_{n}}\right|>0 .
$$

This is a condition which is necessary for solving many interpolation problems. See [2], [13] and [14] for example.

Denote by $B V_{1}$ all sequences $\left\{a_{n}\right\}_{n=1}^{\infty}$ such that $\Sigma_{1}^{\infty}\left|a_{n+1}-a_{n}\right|<\infty$. $B V_{1}$ is a Banach space with norm

$$
\left\|\left\{a_{n}\right\}_{n=1}^{\infty}\right\|=\left|a_{1}\right|+\sum_{1}^{\infty}\left|a_{n+1}-a_{n}\right| .
$$

We also let $B_{1}$ denote the Banach algebra of all analytic functions in $D$ whose derivative belongs to $H^{1}(D)$. The norm on $B_{1}$ is given by $N(f)=$ $\|f\|_{D}+\left\|f^{1}\right\|_{1}$.

If $S=\left\{z_{n}\right\}_{n=1}^{\infty} \subset D$ satisfies (C) and converges to 1 non-tangentially, (which means that $\left|1-z_{n}\right| \leq \lambda\left(1-\left|z_{n}\right|\right.$ ), $n=1,2, \ldots$ for some $\lambda>0$ ) Vinogradov proved that $\left.B_{1}\right|_{s}=B V_{1}$.

Our result is:

THEOREM 4. Assume $S=\left\{z_{n}\right\}$ satisfies (C) and converges to 1 nontangentially. For each $\left\{a_{n}\right\} \in B V_{1}$ there exists $f$ analytic in $\mathbf{C} \backslash\{1\}$ interpolating $\left\{a_{n}\right\}$ at $\left\{z_{n}\right\}$ such that $f$ is bounded in $\{w:|1+w| \leq 2\}$ and $\left.f^{\prime}\right|_{D} \in$ $H^{1}$.

Proof. We first prove that each $\left.g \in B_{1}\right|_{s}$ extends to a bounded analytic function $h$ in $\{w:|1+w|<2\}$ with $\left.h^{\prime}\right|_{D} H^{\prime}$.

Define $\phi(z)=(1+z) / 2, z \in \mathbf{C}$. By the theorem of Vinogradov it is sufficient to show that $\left\{\Phi\left(z_{n}\right)\right\}_{n=1}^{\infty}$ satisfies (C). (Observe that $f \in B_{1} \Rightarrow h=$ $\left.f_{0} \phi B_{1}\right)$. Clearly $w_{n}=\phi\left(z_{n}\right) \rightarrow 1$ non-tangentially.

By a recent result of Kam-Fook Tse [12], Theorem 1, page 352, it is sufficient to find $t>0$ such that 


$$
\inf _{i, j}\left|\frac{w_{i}-w_{j}}{1-\bar{w}_{j} w_{i}}\right| \geq t
$$

Since $\left\{z_{n}\right\}$ satisfies (C) this is easy and we omit it. But then we can deduce Theorem 4 from Theorem 2 .

Final remarks. We now give the example showing that (iii) in Theorem 1 may fail. Let $R=\{z=x+i y: 0<x<1,-1<y<1\}$ and define $R_{n}=\left\{z=x+i y: 2^{-3 n-2} \leq x \leq 2^{-3 n-1},|y|>\varepsilon_{n}\right\}$ for $n=1,2, \ldots$ where $\left\{\varepsilon_{n}\right\}$ is a sequence to be specified. Let $I_{n}=\left(2^{-3 n-4}, 2^{-3 n-2}\right)$ and choose a finite set of points $S_{n} \subset I_{n}$ with the following property: If $f$ is an analytic function vanishing on $S_{n}$ and bounded by one on the rectangle $D_{n}$ $=\left\{z=x+i y: x \in I_{n},|y|<1\right\}$ then $\left|f\left(2^{-3 n-3}+i y\right)\right|<n^{-1}$ if $|y|<1-$ $n^{-1}$. Let now $U=R \backslash \cup_{n} R_{n}$ and $S=\cup_{n} S_{n}$. Clearly $\bar{S} \backslash S=\{0\}$ and if $f$ $\in H^{\infty}(U)$ then $f\left(2^{-3 n}+i y\right) \rightarrow 0$ as $n \rightarrow \infty$ if $|y|<1$. It follows that $\Pi\left(\tilde{S}^{\tilde{S}}\right)$ includes the segment $\{x=0,-1<y<1\}$. It only remains to show that $\left\{\varepsilon_{n}\right\}$ can be choosen such that $S$ is the zero set of a nonzero function $h$ in $H^{\infty}(U)$. Let $g_{n}$ correspond to $S_{n}$ and $D_{n}$ in the same way as $g$ corresponded to $S$ and $V$ in the proof of Theorem 1 . Define $g_{n} \equiv 1$ outside $D_{n}$. Using Vitushkin's scheme for approximation ([6], page 210) it is easy to find functions $h_{n}$ such that $h_{n} g_{n}$ is analytic near the endpoints of $I_{n}, h_{n}$ is analytic where $g_{n}$ is and $\left|1-h_{n}(z)\right|<2^{-n}$ if dist $\left(z, I_{n}\right)$ is less than $n^{-1} 2^{-3 n}$. (Approximate $\log \left(g_{n}\right)$ near the endpoints of $I_{n}$ and take exponentials and call this function $h_{n}$.) Moreover $\sup \left\{\left|h_{n}(z)\right|, z \in C\right\} \leq A$ where $A$ is an absolute constant. It follows that the infinite product consisting of all the factors $h_{n} g_{n}, n=1,2, \ldots$ is analytic in $\cup_{n} D_{n}$ and in a neighbourhood of the closure of $I_{n}$ for $n=1,2, \ldots$. So if the $\varepsilon_{n}$ tend sufficiently rapidly to zero, $h$ will be in $H^{\infty}(U)$ and $S$ will be zero set of $h$.

The author wishes to thank the referee for some very useful criticism and for the example related to Theorem 1. He also wishes to thank Dr. A. M. Davie for some useful communication.

\section{REFERENCES}

1. E. J. Akutowicz and L. Carleson, The analytic continuation of interpolatory functions, J. Analyse Math., 7 (1959-60).

2. L. Carleson, An interpolation problem for bounded analytic functions, Amer. J. Math., 80 (1958), 921-930.

3. J. Detraz, Algebres de Fonctions analytique dans le disque, Ann. Sci. Ecole Norm. Sup., 3 (1970), 313-352.

4. P. L. Duren, Theory of $H^{P}$-Spaces, Academic Press, 1970.

5. J. P. Earl, On the interpolation of bounded sequences by bounded functions, J. London 
Math. Soc., (2), 2 (1970), 544-548.

6. T. W. Gamelin, Uniform Algebras, Prentice Hall, Englewood Cliffs, N. J. (1967).

7. , The Shilov boundary of $H^{\infty}(U)$, To appear.

8. E. A. Heard and J. H. Wells, An interpolation problem for subalgebras of $H^{\infty}$, Pacific J. Math., 28 (1969), 543-553.

9. K. Hoffman, Banach Spaces of Analytic Functions, Prentice Hall, Englewood Cliffs. N. J. 1962.

10. D. Sarason, Weak-star density of polynomials, J. für die reine und angewandte mathematik, (1972).

11. A. Stray, Approximation and interpolation, Pacific J. Math., 40 (1972).

12. Kam-Fook Tse, Non-tangential interpolating sequences and interpolation by normal functions, Proc. Amer. Math. Soc., 29 (1971).

13. S. A. Vinogradov, Interpolation and zeros of power series with a sequence of coefficients from $L^{p}$, Soviet Math. Dokl., 6 (1965), 57.

14. , Paley features and Rudin Carleson interpolation theorems for some classes of analytic functions, Soviet Math. Dokl., 9 (1968), 111-114.

15. J. Wermer, Seminar über Funktionen Algebren, Springer Verlag, Berlin (1964).

Received November 21, 1972 and in revised form October 10, 1973.

UNIVERSITY OF EDINBURG 



\section{PACIFIC JOURNAL OF MATHEMATICS}

\section{EDITORS}

RICHARD ARENS (Managing Editor)

University of California

Los Angeles, California, 90024

\section{R. A. BEAUMONT}

University of Washington

Seattle, Washington 98105

\section{J. DugunDJI}

Department of Mathematics University of Southern California Los Angeles, California 90007

D. Gillbarg AND J. MilgRAM

Stanford University Stanford, California 94305

\section{ASSOCIATE EDITORS}

E. F. BECKENBACH

B. H. NEUMANN

SUPPORTING

UNIVERSITY OF BRITISH COLUMBIA

UNIVERSITY OF CALIFORNIA

MONTANA STATE UNIVERSITY

UNIVERSITY OF NEVADA

NEW MEXICO STATE UNIVERSITY

OREGON STATE UNIVERSITY

UNIVERSITY OF OREGON OSAKA UNIVERSITY
F. WOLF

K. YosHIDA

INSTITUTIONS

UNIVERSITY OF SOUTHERN CALIFORNIA

STANFORD UNIVERSITY

UNIVERSITY OF TOKYO

UNIVERSITY OF UTAH

WASHINGTON STATE UNIVERSITY

UNIVERSITY OF WASHINGTON

AMERICAN MATHEMATICAL SOCIETY

NAVAL WEAPONS CENTER

The Supporting Institutions listed above contribute to the cost of publication of this Journal, but they are not owners or publishers and have no responsibility for its content or policies.

Mathematical papers intended for publication in the Pacific Journal of Mathematics should be in typed form or offset-reproduced, (not dittoed), double spaced with large margins. Underline Greek letters in red, German in green, and script in blue. The first paragraph or two must be capable of being used separately as a synopsis of the entire paper. Items of the bibliography should not be cited there unless absolutely necessary, in which case they must be identified by author and Journal, rather than by item number. Manuscripts, in duplicate if possible, may be sent to any one of the five editors. Please classify according to the scheme of Math. Rev. Index to Vol. ${ }^{39}$. All other communications to the editors should be addressed to the managing editor, or Elaine Barth, University of California, Los Angeles, California, 90024.

100 reprints are provided free for each article, only if page charges have been substantially paid. Additional copies may be obtained at cost in multiples of 50 .

The Pacific Journal of Mathematics is issued monthly as of January 1966. Regular subscription rate: $\$ 72.00$ a year (6 Vols., 12 issues). Special rate: $\$ 36.00$ a year to individual members of supporting institutions.

Subscriptions, orders for back numbers, and changes of address should be sent to Pacific Journal of Mathematics, 103 Highland Boulevard, Berkeley, California 90708.

\section{PUBLISHED BY PACIFIC JOURNAL OF MATHEMATICS, A NON-PROFIT CORPORATION}

Copyright (C) 1974 by Pacific Journal of Mathematics

Manufactured and first issued in the U.S.A. 


\section{Pacific Journal of Mathematics}

\section{Vol. 54, No. $2 \quad$ June, 1974}

John Edward Coury, Walsh series with coefficients tending monotonically to

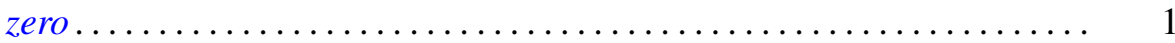

Patrick Michael Fitzpatrick and Walter Volodymyr Petryshyn, Fixed point theorems for multivalued noncompact acyclic mappings ............

Irving Leonard Glicksberg, More on Phragmén-Lindelöf for function

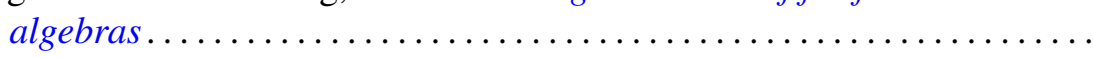

Adilson Goncalves, Structural constants. II .................. 39

Richard P. Gosselin, Closure theorems for affine transformation groups .... 53

Ralph Peter Grimaldi, Baer and UT-modules over domains ........... 59

Edward Grossman, On the prime ideal divisors of $\left(a^{n}-b^{n}\right) \ldots \ldots \ldots \ldots . \ldots 73$

A. Hedayat and Ester Seiden, On the theory and application of sum composition of Latin squares and orthogonal Latin squares.......... .

Gerald L. Itzkowitz, Continuous measures, Baire category, and uniform continuity in topological groups ......................... 115

Francis Masat, Right simple congruences on a semigroup ............ 127

Robert Harvey Oehmke, Right congruences and semisimplicity for Rees matrix semigroups..................................

Qazi Ibadur Rahman and Jan Stankiewicz, Differential inequalities and local valency . . . . . . . . . . . . . . . . . . . . . . . . . . . . . . . . . . . . . 165

William John Reed, Random points in a simplex ................ 183

Mohan S. Shrikhande, Strongly regular graphs and group divisible

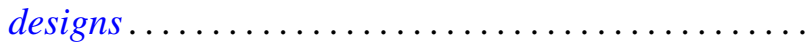

Zahava Shmuely, The structure of Galois connections ... . .

Robert C. Shock, Dual generalizations of the Artinian and Noetherian

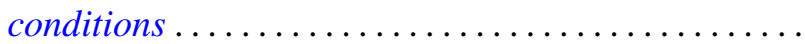

Arne Stray, Approximation and interpolation for some spaces of analytic functions in the unit disc ........................

Eldon Jon Vought, Monotone decompositions into trees of Hausdorff continua irreducible about a finite subset ............

James Wirth, The mapping cylinder axiom for WCHP fibrations ... 263

Gordon S. Woodward, Invariant means and ergodic sets in Fourier analysis... 